CP, 2021, Vol.10 - No20, pp. 89/100, ISSN 2014-6752. Girona (Catalunya). Universitat de Girona. ACHARKI, Z.: Docencia universitaria en entornos virtuales de aprendizaje. Recibido: 24/02/2021 - Aceptado: 07/06/2021

\title{
Docencia universitaria en entornos virtuales de aprendizaje
}

\author{
Dr./ Zoubair ACHARKI \\ (https://orcid.org/0000-0002-5108-0237) \\ Profesor universitario \\ Departamento de Literatura Hispánica \\ Universidad Abdelmalek Essaadi \\ Tetuán, Marruecos \\ z.acharki@uae.ac.ma
}

\section{Resumen}

Este artículo aborda las posibilidades del uso de los entornos virtuales de aprendizaje implementados desde la propagación del Covid-19 en el ámbito universitario y sus implicaciones en la redefinición de los roles docentes y los procedimientos metodológicos. El interés de esta investigación nace de la necesidad de incorporar soluciones tecnológicas y aprovechar el potencial de la enseñanza virtual para el desarrollo de los procesos educativos desde un enfoque constructivista. En este marco, el objetivo de la investigación es mostrar la importancia que tiene la integración efectiva de los Entornos virtuales de aprendizaje para el desarrollo de un aprendizaje significativo y determinar las posibilidades que ofrece la aplicación didáctica de esta modalidad de enseñanza para mejorar y ampliar el proceso de construcción del conocimiento. Mediante un enfoque cualitativo, la investigación está basada en una revisión documental de la literatura que resalta la utilidad de valorar los entornos virtuales como una poderosa herramienta didáctica para la construcción de escenarios de enseñanza basados en la modalidad virtual. Asimismo, se ex-

\section{Abstract}

This paper addresses the possibilities of using the Virtual Learning Environments implemented since the spread of Covid-19 in the university sphere and its implications in the redefinition of roles of teachers and methodological procedures. The interest of this research stems from the need to incorporate technological solutions and take advantage of the potential of virtual teaching for the development of educational processes from a constructivist approach. In this framework, the objective of the research is to show the importance of the effective integration of Virtual Learning Environments for the development of meaningful learning and to determine the possibilities offered by the didactic application of this teaching modality to improve and expand the knowledge construction process. Using a qualitative approach, the research is based on a review of the literature that sheds light on the usefulness of evaluating Virtual Learning Environments as a powerful didactic tool for the construction of teaching scenarios based on the virtual modality. Likewise, the new roles of teachers that university education implies in this educational mo- 
ponen los nuevos roles docentes que implica la enseñanza universitaria en este modelo educativo. Se concluye que el uso de los Entornos virtuales de aprendizaje demanda nuevos roles docentes (acompañante, tutor, diseñador de escenarios educativos y motivador de aprendizajes) y posibilita un aprendizaje constructivista a partir de distintos tipos de interactividad (individual, instrumental y social).

Palabras clave: construcción del conocimiento; entornos virtuales de aprendizaje; pandemia; procedimientos metodológicos; rol docente. del are exposed. It is concluded that the use of Virtual Learning Environments demands new teacher's roles (counselor, designer of educational scenarios and learning motivator) and enables constructivist learning from different types of interactivity (individual, instrumental and social).

Keywords: knowledge construction; virtual learning environments; pandemic; methodological procedures; teacher's role.

\section{Introducción}

Desde la irrupción de la pandemia del Covid-19 y la suspensión de las clases presenciales, los docentes del ámbito universitario se han visto en la necesidad de explorar las posibilidades de la enseñanza mediada por la virtualidad a fin de darle un nuevo sentido a los procesos didácticos.

Este movimiento hacia la virtualidad impuesto por la pandemia del COVID-19 está resultando un desafío inevitable que obliga a actuar incluso a aquellos actores que son más resistentes a una mayor apropiación de la cultura digital. Las instituciones tradicionales ahora deben concebir las experiencias de aprendizaje remoto como un aspecto central del proceso integral de formación. Este enclaustramiento global también representa una gran oportunidad para quienes promovemos que los docentes universitarios dejen de ser meros divulgadores de contenidos y se transformen en diseñadores de experiencias de aprendizaje (Cobo y Kuklinski, 2020, p.8).

Ante esta nueva situación, el gran reto al que se enfrenta el profesor universitario gira en torno a su capacidad de generar planes de acción que promuevan ambientes de aprendizaje significativo y estimulen nuevas formas de pensar con relación a la manera de adquirir conocimientos en un entorno mediado por la tecnología.

Dentro de las nuevas modalidades de enseñanza que han emergido con mayor fuera a raíz de la actual situación de crisis sanitaria se encuentran los entornos virtuales de aprendizaje (EVA) debido al gran abanico de posibilidades que ofrecen para la gestión y desarrollo del proceso educativo.

Una de las principales características de los EVA es la capacidad de desarrollar un aprendizaje colaborativo centrado en la actividad del estudiante y la interacción para la construcción de conocimientos. En este contexto, la función del estudiante supera la mera asimilación de contenidos para desarrollar un aprendizaje activo y flexible basado en la realización de diversas actividades a través de la interacción con el entorno, desde el simple tratamiento de textos hasta la interacción con otros sujetos, pasando por 
la gestión de la información y el conocimiento. Ello demanda el replanteamiento del quehacer docente y la puesta en práctica de un conjunto de concepciones y enfoques novedosos orientados hacia la actividad del estudiante, la comunicación y la colaboración (gestión del conocimiento, trabajo en equipo, acción docente mediada por EVA, etc.).

En este marco de ideas, el siguiente artículo tiene como objetivo reflexionar sobre las posibilidades del uso de los EVA en el ámbito universitario y analizar las implicaciones de su integración para la configuración de nuevos roles docentes y la redefinición de los procedimientos metodológicos. Todo ello en un contexto social en el que las herramientas tecnológicas se imponen hoy más que nunca y que requieren una adecuada reflexión didáctica para desarrollar procesos de construcción de conocimientos.

\section{Metodología}

El artículo es de carácter descriptivo y se basa en la revisión documental de los aportes pedagógicos constructivistas que justifican en el empleo de las herramientas tecnológicas, en concreto, los entornos virtuales de aprendizaje (EVA) en el ámbito universitario. Se trata de comprender, desde un enfoque cualitativo, los fundamentos teóricos y didácticos de la enseñanza virtual con el propósito de concretar planes operacionales de acción susceptibles de guiar el proceso de aprendizaje en la situación actual que vivimos con respecto a la pandemia del Covid-19.

El análisis de contenido se realiza partiendo de las siguientes preguntas de investigación: 1) ¿Qué aportan los entornos virtuales de aprendizaje a la enseñanza universitaria?; 2) ¿Qué implicaciones tiene la implementación de estos nuevos escenarios en la configuración de la práctica docente en el ámbito universitario?; y 3) ¿Cuáles son los principales procedimientos metodológicos que requiere la enseñanza virtual para lograr de un aprendizaje significativo?

\section{Posibilidades del uso de EVA en el ámbito universitario}

En términos generales, un EVA es un espacio que integra un conjunto de recursos y herramientas tecnológicas que posibilitan el desarrollo del aprendizaje y favorecen la comunicación (síncrona y asíncrona) entre los principales actores educativos. En este espacio virtual, el docente suele plantear propuestas didácticas para llevar a cabo el proceso de enseñanza y aprendizaje. Andino y Sánchez (2017) definen un EVA como:

Un espacio de comunicación que hace posible, la creación de un contexto de enseñanza y aprendizaje en un marco de interacción dinámica, a través de contenidos culturalmente seleccionados y elaborados y actividades interactivas para realizar de manera colaborativa, utilizando diversas herramientas informáticas soportadas por el medio tecnológico, lo que facilita la gestión del conocimiento, la motivación, el interés, el autocontrol y la formación de sentimientos que contribuyen al desarrollo personal (p.9).

En su acepción técnica, los EVA hacen referencia a:

un entorno que permite el acceso a través de navegadores, generalmente con acceso protegido; utiliza servicios y herramientas de la Web; dispone de una interface gráfica e intuitiva; integra diferentes módulos para la gestión formación, organización de cursos, calendario, recursos educativos digitales, gestión de actividades, seguimiento y evaluación del aprendizaje; permite asignar privilegios según el rol del usuario, posibilita la comunicación e interacción entre los estudiantes- docente-tutor y grupo, dentro de la comunidad y entre comunidades y con las redes sociales, permite realizar e implementar diferentes actividades e incorpora recursos para el seguimiento y evaluación del proceso (Fernández, 2011, como se citó en Montenegro y Nodarse, 2017, p.5). 
Por tanto, un EVA es un espacio diseñado exclusivamente para incorporar los recursos y las funcionalidades, tanto de comunicación como de información y de gestión, que permiten la interacción didáctica y facilitan el trabajo del profesor y los estudiantes. Si bien los entornos virtuales suelen presentar diferencias en cuanto a la configuración de su interfaz, las funcionalidades, la organización de la docencia y el aprendizaje, los recursos disponibles y herramientas de comunicación suelen ser similares en su gran mayoría.

El potencial de los EVA ha sido explorado por muchos investigadores y puesto en práctica por muchos docentes en todos los niveles educativos, demostrando que los estudiantes son capaces de expresarse y demostrar los nuevos conocimientos que han adquirido. En esta línea, Escartín (2005) afirma respecto a los entornos virtuales:

La tecnología de la Realidad Virtual (RV) ha sido ampliamente señalada como un desarrollo tecnológico importante que puede apoyar al proceso de enseñanza - aprendizaje de varias formas. Algunas de sus excepcionales capacidades son la posibilidad de permitir a los estudiantes la visualización de conceptos abstractos, observar eventos a escalas atómicas o planetarias, o visitar ambientes e interactuar con eventos que la distancia, el tiempo o los factores de seguridad los hacen completamente inalcanzables en condiciones reales. Las actividades educativas que pueden ser soportadas por estas capacidades de la RV conducen a la opinión actual de que los estudiantes podrán alcanzar un mejor dominio, retención y generalización de los nuevos conocimientos en la medida en que se involucren activamente en la construcción de ese conocimiento en situaciones de aprendizaje activo. (p.1)

Las posibilidades que ofrecen los EVA fomentan la participación activa y el interés de los estudiantes para el aprendizaje y favorecen una interpretación personal y una mayor asimilación de los contenidos mediante la interacción con el entorno. Esta interacción puede desarrollarse de forma individual mediante la gestión de la información por parte del estudiante (exploración, creación y publicación), pero también se da una interacción social en la que los estudiantes interactúan entre sí y con el profesor.

La interacción entre actores puede promoverse a través de la construcción colectiva del conocimiento para opinar, exponer y debatir ideas desde la diversidad de pensamientos. Además de la interacción entre actores, existe la interacción con los contenidos, es decir, la interacción estudiante-contenidos; esta se relaciona con la competencia para organizar, reconocer, aplicar y difundir la información. (Gómez, Peralta, Herrera, Corrales y Angarita, 2016, p.9)

Desde el punto de vista didáctico, un EVA es un espacio que, por una parte, permite a cada estudiante interactuar, de forma individual, con la información e interpretarla a partir de sus conocimientos previos y progresar a su ritmo para que finalmente sea capaz de construir una representación personal de los nuevos conocimientos. Además, la interacción del estudiante con el contenido posibilita el desarrollo del aprendizaje autorregulado mediante el cual se genera en los estudiantes un estilo propio de implicación en la resolución de tareas, estableciendo sus propias metas, planteando sus propias estrategias para evaluar el grado de cumplimiento de los objetivos, procesando información y encontrando recursos para aprender (Izquierdo, 2010).

Por otra parte, la construcción del conocimiento se desarrolla también de forma colectiva mediante la colaboración entre todos los participantes del curso a través de herramientas de comunicación como 
foros de discusión, chat y correo electrónico. Esto implica varias posibilidades de comunicación. En primer lugar, el profesor puede comunicar cuestiones académicas relevantes o publicar guías e información fundamental para seguir el curso. En segundo lugar, existe la posibilidad de disponer de un espacio de encuentro e intercambio de opiniones y experiencias sobre los temas estudiados de modo asincrónico, donde el profesor y los estudiantes pueden plasmar las ideas principales de los temas abordados, compartir enlaces de interés, enviar comentarios, formular preguntas, plantear dudas, etc.

De esta forma, las diferentes interacciones que posibilitan los EVA permiten un aprendizaje colaborativo y aumentan la participación activa de los estudiantes entre sí y con el profesor mediante el intercambio de información y el diálogo a través de las herramientas de comunicación, debates y actividades colaborativas, y estimulan el aprendizaje autónomo frente a la dependencia del profesor en el modelo tradicional de enseñanza.

Por todo ello, la implementación de los EVA implica una reconsideración en la adquisición del conocimiento no como hecho individual sino social en el sentido de que no se construye de manera individual sino mediante la resolución de problemas junto con los demás individuos dentro de un contexto sociocultural que da significado al propio conocimiento. En este sentido, el estudiante se convierte en constructor de sus aprendizajes mediante la interacción con el entorno que le proporciona el profesor. Por consiguiente, los entornos virtuales se conciben como una herramienta tecnológica mediadora de las relaciones entre profesores y estudiantes en la que prima la interacción y las relaciones humanas sobre otros aspectos como la mera información.

Por otra parte, la psicología cognitiva plantea el sistema de navegación de los entornos virtuales de aprendizaje como un modelo próximo a la estructura semántica de la mente humana en el sentido de que se define una estructura de relaciones en red y unos nodos de información que interactúan en forma dinámica (García, E., 2015).

En este sentido, un ambiente hipertextual de aprendizaje constituye una base de datos asociativa que sigue la tendencia natural de la selección de la información por asociación. Si bien el docente es quien determina los múltiples enlaces, el estudiante tiene la posibilidad de elegir su propio itinerario siguiendo una lógica personal.

La posibilidad de navegar por un entorno de aprendizaje con una estructura abierta para la resolución de problemas permite que estudiantes con diferentes niveles de conocimientos puedan seguir caminos diferentes en su trabajo de acuerdo con sus propias necesidades. La estructura no lineal de un entorno virtual permite que tanto el usuario experto como un inexperto puedan aprender y valerse del mismo sistema informático. Además resulta altamente ventajoso que el usuario pueda elegir distintos caminos, puesto que agiliza el proceso de recuperación de la información necesitada y favorece la asimilación de la información al desarrollarse este proceso de modo más activo (García, E., 2015).

En resumidas cuentas, los entornos virtuales permiten nuevas formas de aprender centradas en la actividad del estudiante (asociar información, crear relaciones entre distintos contenidos, añadir y eliminar información, etc.) y abren múltiples posibilidades de interactuar e influir sobre los demás y realizar transformaciones significativas en los procesos de enseñanza y aprendizaje sin tener que establecer un contacto social directo. 
Evidentemente, conseguir esto requiere de una adecuada capacitación para el uso de EVA. De ahí que tanto los profesores como los estudiantes deberán poseer destrezas mínimas que le ayuden a comprender las nuevas herramientas tecnológicas y su uso, ya que el éxito de la implementación de estos entornos, es decir, su capacidad para construir, representar y generar conocimientos, dependerá en gran medida de la capacitación de los sujetos implicados directamente para interaccionar con las mismas.

Además de contar con un conocimiento de los principios básicos de las herramientas tecnológicas, la implementación de los EVA en el ámbito educativo demanda la aplicación de un adecuado saber didáctico que permita la consecución de los objetivos de la enseñanza de una determinada materia. Igualmente importante resulta la dotación de las universidades de los recursos e instrumentos necesarios para afrontar los retos de la sociedad de la información y saber interactuar con ellos.

\section{Roles docentes en los EVA en el ámbito universitario}

Las posibilidades de los escenarios virtuales en el ámbito universitario conducen inevitablemente a un cambio de paradigma en la acción docente para desarrollar las interacciones que se ponen en juego en esta nueva modalidad educativa. A este respecto, Martínez Martín (2008) señala:

Conviene que tal integración comporte un auténtico cambio en el profesorado tanto en la manera de comprender tales procesos como en la de disponer los contenidos de aprendizaje, de forma que la integración de tecnologías no sea un simple cambio de escenario en el espacio de aprendizaje universitario -menos presencial, más virtual y más centrado en el aprendizaje autónomo del estudiante- y comporte realmente una docencia más eficiente y más aprendizaje en el estudiante y de mayor calidad. (p.228)

La toma de conciencia del profesor de sus nuevos roles docentes ejerce una influencia determinante en la forma de desarrollar sus prácticas de enseñanza ya que estos roles están interrelacionados con otros elementos configuradores de su labor docente como los objetivos y contenidos didácticos que persigue la docencia en una materia determinada, la concepción sobre las metodologías didácticas y las modalidades y criterios de evaluación.

Uno de los roles docentes fundamentales en la enseñanza mediada por EVA es el de ser acompañante, guía y facilitador del aprendizaje. Por una parte, el acompañamiento del estudiante se basa en la interacción y la colaboración entre el profesor y los estudiantes a través del intercambio de ideas, opiniones, reflexiones, experiencias, fuentes de información, etc. Por otra parte, el rol de acompañante implica organizar y gestionar los diferentes elementos curriculares necesarios para facilitar al estudiante la construcción del conocimiento (Bautista, G., Borges, F. Y Forés, A. 2006). En este sentido, el profesor debe proponer al estudiante la forma de abordar los contenidos de la asignatura atendiendo sus intereses, guiarle hacia la resolución de los problemas o dudas, formular criterios de evaluación, etc.

El acompañamiento implica también saber organizar y dinamizar actividades individuales y grupales en la modalidad virtual mediante metodologías activas y la selección previa de contenidos adecuados al perfil y a los intereses de los estudiantes. En esta línea, Torrecilla (2005) aclara:

Siendo el ciberespacio un espacio común de redes de aprendizaje en el cual grupos de personas se conectan, donde - desde un ámbito educativo- profesores y educadores pueden crear entornos efectivos de aprendizaje y acompañarán a los estudiantes para que trabajen juntos en la produc- 
ción de conocimientos. La red ofrece la oportunidad de interactuar dando opción a gestionar grupos de trabajo alrededor de comunidades educativas o el desarrollo de actividades centradas en el trabajo colaborativo. (p.32)

De este modo, el docente se convierte en un animador de la inteligencia colectiva del grupo del que se responsabiliza, dirigiendo su actuación a la dinamización de espacios de debate o de ayuda colectiva, así como espacios para investigar y difundir los conocimientos.

Otro de los roles que desempeña el docente en un EVA se refiere a la acción tutorial, orientando a los estudiantes para desenvolverse en su propio aprendizaje. La función tutorial consiste en facilitar a los estudiantes los contenidos curriculares y acompañarles en su proceso de aprendizaje, pero supone también avituallarles con las estrategias y recursos necesarios para la gestión del tiempo, la realización de las actividades y el intercambio de conocimientos en un contexto social.

El proceso de tutorizar es un proceso de apoyo y asistencia que el profesor debe llevar a cabo con el alumno, para que pueda integrarse en el nuevo entorno formativo tanto a nivel técnico como social. Este proceso permite además que el alumno comprenda los contenidos que se le presenten a través de nuevos soportes, pueda integrarse socialmente en el grupo de formación y en la acción formativa en sí misma, evitando el abandono producido por el aislamiento. (Aguilera y Noris, 2014, p.52)

En la actual situación presentada por la pandemia, impregnada de estrés y agobio, uno de los retos más importantes para el docente universitario consiste en saber motivar a sus estudiantes para que aprendan y diseñar estrategias y acciones de motivación. Por lo tanto, la tutorización en la enseñanza requiere también que el profesor se convierta en un motivador de aprendizajes en el sentido de que sea capaz de despertar el deseo de aprender de sus estudiantes para que sean sujetos activos de su propio aprendizaje. Esto demanda un gran esfuerzo para elaborar propuestas de aprendizaje atractivas que estimulen al estudiante para desarrollar sus aprendizajes, fomenten la comunicación, el intercambio de conocimientos y le ayuden en la selección, análisis y adquisición de la información así como el desarrollo de competencias, actitudes y valores.

Conseguir que los estudiantes estén motivados y muestren interés por aprender precisa además de la instauración de una didáctica específica. A través de la reflexión docente sobre la propia acción y el diseño de las estrategias didácticas adecuadas, los profesores deben incentivar el interés de los estudiantes por las diferentes actividades a realizar, mostrar su utilidad, explicitar las estrategias adecuadas para resolverlas y mostrar la importancia que esos conocimientos y procedimientos tienen para su formación integral como estudiantes universitarios.

Los contenidos propuestos, a su vez, deben tomar en consideración los factores motivacionales de cada estudiante ya que la falta de consideración de las variables afectivas de los estudiantes a la hora de seleccionar los contenidos puede convertirse en un obstáculo para el buen desarrollo de la acción didáctica.

Otro rol docente importante en un EVA hace referencia al diseño y organización de los materiales y recursos didácticos para generar conocimientos y desarrollar la interacción cognitiva. Esto supone elaborar propuestas didácticas concretas tomando en consideración los contenidos de la asignatura, la didáctica de la misma, los intereses y características de los estudiantes y las herramientas que incluyen los entornos virtuales. En este sentido, el docente debe articular entre sus competencias digitales, pedagógicas y 
de gestión para crear entornos de aprendizaje susceptibles de acompañar al estudiante en su proceso de integración de los conocimientos adquiridos y el desarrollo de sus competencias.

En definitiva, el desempeño de todos estos roles implica pasar de una concepción del profesorado entendido como transmisor de conocimiento y experto en la materia a otro perfil docente, orientador y mediador de la actividad educativa. Para ello, los profesores deberán aprovechar las ventajas que ofrecen los entornos virtuales para favorecer nuevos espacios de aprendizaje con más énfasis en el proceso intelectual del estudiante, pero sobre todo, en cuanto a su capacidad de desarrollar un aprendizaje sustentado en el intercambio de ideas, reflexiones, experiencias y fuentes de información.

\section{Procedimientos metodológicos en los EVA}

La metodología docente permite concretar el diseño curricular mediante la planificación, desarrollo y evaluación de los contenidos curriculares así como la intervención didáctica para su concreción. Por tanto, la metodología constituye aquel elemento del currículum que especifica las actividades y experiencias más adecuadas para capacitar al estudiante para aprender los contenidos y desarrollar las competencias necesarias.

En los entornos virtuales, la metodología docente debe favorecer procesos dinámicos a través de propuestas didácticas que militen a favor de la adquisición de competencias y la formación de estudiantes capaces de acceder al conocimiento y generar otro nuevo mediante su capacidad investigativa y su interacción en la virtualidad.

No se trata tan sólo de impartir clases sino de diseñar una arquitectura coherente de experiencias autónomas de aprendizaje que deberían resultar significativas para el estudiante con el fin de promover el intercambio de información, la comunicación y la colaboración entre los principales actores educativos. Por ello, el docente debe favorecer el desarrollo de aprendizajes flexibles y variados que ayuden al estudiante a realizar diversas tareas mediante medios interactivos (interacción cognitiva y social, gestión de la información, etc.).

Por consiguiente, los procedimientos metodológicos constituyen un elemento clave para el desarrollo de procesos significativos de aprendizaje en un EVA. El docente debe saber compaginar las metodologías tradicionales con otras metodologías más dinámicas, basadas en la construcción del conocimiento, la comunicación y la colaboración, desde las cuales la práctica docente es percibida en tanto que búsqueda constante de ideas, enfoques y metodologías y no como un proyecto definitivo y acabado.

Como ocurre en un entorno presencial, en un EVA, existen tres pautas de actuación docente que ha de tenerse en cuenta a la hora de desarrollar la actividad docente. Estas pautas se refieren a las tres fases del proceso de enseñanza y aprendizaje, estos es: al comienzo de las actividades de aprendizaje (fase inicial), durante el trabajo (fase de desarrollo) y finalmente en la evaluación de los aprendizajes (fase final o de evaluación) (Bautista, G., Borges, F. Y Forés, A. 2006).

La fase inicial es un momento fundamental para captar la atención del estudiante, despertar su curiosidad, conectar sus conocimientos previos con los nuevos contenidos, mostrar la relevancia de esos conocimientos y las destrezas que se van a adquirir y crear las condiciones óptimas para estimular su interés para el aprendizaje. Para ello, el docente puede organizar un encuentro virtual inicial a través del chat de la plataforma para diagnosticar el nivel del grupo en el área del que trata el curso y explicar los objetivos que se pretenden alcanzar a lo largo del mismo. 
En estos primeros momentos el docente deberá también orientar al estudiante con respecto a las normas de funcionamiento del espacio virtual en el que se desarrollará el proceso educativo. Por ello, es importante que el estudiante tenga disponible desde el inicio del curso un corpus de información que le oriente en su proceso de aprendizaje.

Una forma de orientar a los estudiantes desde el primer momento del proceso de aprendizaje en un EVA es a partir del diseño de guías didácticas en las que se explican los objetivos del curso, la metodología, los materiales y recursos utilizados, el temario, el desarrollo de las actividades, los trabajos a elaborar, los criterios de evaluación, etc. Además, el profesor puede elaborar guías de aprendizaje para acompañar a los estudiantes a lo largo del proceso de construcción de su conocimiento (mapas conceptuales, esquemas, resúmenes, etc.). Estos documentos de carácter didáctico están destinados a facilitar la planificación personal del estudiante, mostrar ejemplos prácticos, proponer actividades complementarias, sintetizar y organizar los contenidos

En la fase de desarrollo el profesor debe llevar a cabo propuestas rigurosas de trabajo encaminadas a la consecución de los objetivos del curso. En esta fase, el proceso de aprendizaje puede desarrollarse a partir de una comunicación fluida y frecuente entre los estudiantes y el profesor o bien a partir del uso de materiales didácticos para el seguimiento del curso.

En el primer caso, la interacción entre los principales actores educativos es esencial ya que no existen encuentros físicos entre ellos, por lo que es importante que el profesor haga uso de una gran variedad de estrategias comunicativas para fomentar y consolidar la participación activa de los estudiantes y lograr que se sientan parte de un grupo cohesionado (Bautista, G., Borges, F. Y Forés, A. 2006).

En el segundo caso, es fundamental la elaboración de actividades orientadas hacia la participación y colaboración de los estudiantes. Estas actividades deben tener en cuenta tanto las posibilidades de interacción de la plataforma virtual como los diferentes procesos que realiza el estudiante a la hora de aprender (actividades de presentación, de producción, de búsqueda y selección de la información, de síntesis, debates, trabajos colaborativos, aprendizaje por problemas, etc.).

Existe una gran variedad de actividades que pueden llevarse a cabo en entornos virtuales y que permiten sustituir el modelo tradicional de las clases magistrales por otro tipo de prácticas más dinámicas que buscan no tanto acumular contenidos sino más bien desarrollar capacidades y destrezas que ayuden a los alumnos a aprender de forma autónoma.

Una de las actividades que el profesor puede desarrollar en un EVA es la organización de debates virtuales para intercambiar opiniones y expresar ideas sobre un determinado tema. Este tipo de actividades ofrece a los estudiantes la oportunidad de fortalecer su reflexión y actitud crítica y aprender a comunicar los nuevos conocimientos adquiridos, argumentarlos y utilizarlos para elaborar conocimientos propios. Los debates virtuales pueden desarrollarse a partir de una serie de preguntas sobre un tema o mediante la utilización de diversos soportes multimedia como la visualización de un video, por ejemplo (Bautista, G., Borges, F. Y Forés, A. 2006).

Otro tipo de actividades que pueden llevarse a cabo en los entornos virtuales se refiere a las metodologías de publicación y trabajos colaborativos como el uso de los Weblogs. Este tipo de metodologías se basa en el uso de las herramientas de comunicación para promover la expresión y el intercambio de 
ideas y experiencias personales sobre cualquier tema, creando un entorno más comprometido para el alumno respecto a los temas abordados en la medida en que se le involucra en un trabajo más personal y productivo. Los trabajos realizados se insertan en el weblog en un documento con la finalidad de hacer posible la puesta en común de los diferentes resultados (Barujel, 2005).

Los weblogs ofrecen espacios en los que los alumnos tienen la oportunidad de expresarse y ponerse en contacto unos con otros a pesar de la distancia que les separa físicamente definiendo un aprendizaje que se desarrolla en un contexto social. Además, estas aplicaciones son de gran ayuda para el docente ya que le ofrecen la oportunidad de orientar el trabajo de investigación de sus alumnos a fin de evitar su desorientación en la red y hacer que sean críticos a la hora de buscar y seleccionar la información sobre los temas propuestos en clase, al mismo tiempo que le permiten evaluar el proceso de aprendizaje de sus alumnos.

Respecto a las posibilidades que ofrecen los weblogs, Stefany Hernández (2008) menciona las siguientes funciones:

- responsabilidad y compromiso: el hecho de redactar entradas con fechas específicas en sus blogs, encargarse de actualizarlo e introducir nuevos pensamientos, ideas o artículos crea en los alumnos un concepto de disciplina y de responsabilidad.

- comunicación: los blogs incrementan la comunicación entre los compañeros de clase, profesor e incluso con los familiares. Esta herramienta proporciona a las personas la oportunidad de conectarse, al igual que con las redes sociales, con cualquier integrante del aula, sin que existan barreras de tiempo o espacio.

- tecnología: la tecnología juega un rol importante en cada actividad que realizan actualmente los alumnos. Los blogs motivan el uso de las nuevas construcciones de conocimiento.

- mejora de las habilidades de escritura, gramática y ortografía: al ser leídos por los compañeros de clase, no sólo por el profesor, generan en los alumnos un esfuerzo por presentar su mejor trabajo y esforzarse en la escritura de lo que van a redactar y presentar.

La fase de desarrollo se complementa con otra fase final del proceso que actúa como refuerzo y evaluación de los contenidos desarrollados pero también supone una fase de conclusión y cierre del proceso educativo. Al igual que ocurre en cualquier otra modalidad educativa, la evaluación debe ser un remodelador constante del aprendizaje de los estudiantes. Por ello, el docente debe adoptar un enfoque que compagine tanto la evaluación continua como la evaluación final del proceso de enseñanza y aprendizaje.

La evaluación en un EVA no debe ser una mera reproducción de modalidades evaluativas tradicionales, sino que debe adaptarse a las particularidades de las metodologías de enseñanza, al tipo de contenidos desarrollados y a los objetivos del aprendizaje en un entorno virtual.

En este sentido, a lo largo del proceso de enseñanza y aprendizaje, los métodos evaluativos valorarán aspectos tales como el desempeño del estudiante en una secuencia didáctica, el grado de interés y su participación activa en las distintas actividades, la calidad de los mensajes en las charlas virtuales, etc. Este tipo de evaluación se refiere a una evaluación continua. Por otro lado, en la evaluación sumativa (o final), el profesor valorará el progreso cognitivo y la adquisición de determinadas competencias a través de propuestas concretas en las cuales el estudiante deberá demostrar un dominio de los objetivos de aprendizaje. Entre las propuestas evaluativas destacan: la participación en un debate final moderado por 
el profesor, creación de un producto personal basado en material multimedia, elaboración de proyectos colaborativos, resolución de problemas, actividades de autoevaluación, etc.

\section{Conclusión}

La implementación de estrategias de continuidad de la enseñanza universitaria en entornos virtuales implica necesariamente un cambio en las prácticas docentes para aprovechar al máximo las posibilidades que ofrece esta nueva modalidad educativa. Esto supone concebir los EVA como parte integrante de un proceso que se desarrolla de forma regular en un ambiente de aprendizaje activo y participativo y en el cual las estrategias de enseñanza, los contenidos, los procedimientos y las actividades de aprendizaje se articulan con las herramientas y los recursos de los nuevos entornos.

Por ello, es importante que los docentes asuman los nuevos retos de la enseñanza mediada por entornos virtuales, desarrollen las competencias específicas que requieren estos nuevos escenarios y generen las estrategias necesarias para proporcionar a los estudiantes escenarios reales de aprendizaje a través de actividades de interacción y procedimientos metodológicos que permiten el desarrollo de contenidos, habilidades y competencias imprescindibles para la construcción del conocimiento.

Por otra parte, es preciso señalar que, en este nuevo escenario, no sólo cambian los roles docentes sino que paralelamente se perfilan nuevos roles que deben asumir los estudiantes. El estudiante debe, por tanto, adquirir las competencias básicas que le capaciten para desenvolverse de forma autónoma en un entorno virtual, tales como la búsqueda, selección y asimilación de información, la reflexión y el pensamiento crítico, la capacidad comunicativa, el trabajo en equipo, la resolución de problemas, etc. Por ello, los estudiantes deben desplegar mayores esfuerzos y estar mucho más implicados en su propio proceso de aprendizaje de lo que lo han estado hasta ahora.

Asimismo, la integración efectiva de las TIC en la enseñanza solo puede lograrse si se desarrolla un trabajo en equipo entre los profesores en la planificación y ejecución de los planes de estudios. En este sentido, otro de los grandes retos que tiene por delante la docencia universitaria en el nuevo contexto es lograr unir los esfuerzos del profesorado para que colaboren en la elaboración y puesta en marcha de proyectos consolidados y duraderos de innovación docente, para mejorar la calidad educativa. Se trata de ir transitando de la cultura del individualismo y la falta de coordinación que caracterizaba la relación entre profesores universitarios a otra cultura de cooperación que ayude a mejorar los resultados de aprendizaje a través del intercambio de experiencias.

\section{Referencias bibliográficas}

- $\quad$ Aguilera, Y. y Noris, L. M. (2014). Papel del docente en los entornos virtuales de aprendizaje. Recuperado de http://www.refcale.uleam.edu.ec/index.php/enrevista/article/view/2177

- $\quad$ Andino, M. y Sánchez, H. M. (2017). Entornos virtuales de aprendizaje como apoyo a la enseñanza presencial para potenciar el proceso educativo. Recuperado de https://dialnet.unirioja.es/ servlet/articulo?codigo $=6297476$

- $\quad$ Barujel, A. G. (2005). El uso de weblogs en la docencia universitaria. Recuperado de https:// dialnet.unirioja.es/servlet/articulo?codigo $=1303648$

- $\quad$ BAUTISTA, G., BORGES, F. y FORÉS, A. (2006). Didáctica universitaria en Entornos Virtuales de

Enseñanza-Aprendizaje. Madrid: Narcea. 
- Escartín, E. (2000). La realidad virtual, una tecnología educativa a nuestro alcance. Recuperado de https://dialnet.unirioja.es/servlet/articulo?codigo=1399764

- García, E. (2015). La enseñanza y el aprendizaje en modalidad virtual desde la experiencia de estudiantes y profesores de posgrado de la Universidad Latinoamericana de Ciencia y Tecnología (Tesis doctoral), Universidad Católica de Costa Rica Anselmo Llorente y Lafuente, Maravia-Costa Rica. Recuperado de https://www.aacademica.org/edgar.salgado.garcia/2.pdf

- Gómez, J., Peralta, M., Herrera, M., Corrales, D. y Angarita, M. (2016). Retos y posibilidades del aprendizaje virtual en la educación superior. Recuperado de https://repositorio.uniandes.edu. co/handle/1992/47905

- Hernández, S. (2008). El modelo constructivista con las nuevas tecnologías: aplicado en el proceso de aprendizaje. Recuperado de http://hdl.handle.net/20.500.12579/1210

- Izquierdo, R. (2010): El impacto de las TIC en la transformación de la enseñanza universitaria: repensar los modelos de enseñanza y aprendizaje. Revista Teoría de la Educación. Educación y Cultura en la Sociedad de la Información, 11 (1), 32-68. Recuperado de https://dialnet.unirioja.es/ servlet/articulo?codigo $=3164715$

- Kuklinski, H. y Cobo, C. (2020). Expandir la universidad más allá de la enseñanza remota de emergencia: Ideas hacia un modelo híbrido post-pandemia. Recuperado de https://outliersschool. net/wp-content/uploads/2020/05/Expandir la universidad.pdf

- $\quad$ Martínez, M. (2008). Reflexiones sobre aprendizaje y docencia en el actual contexto universitario. La promoción de equipos docentes. Revista de Educación, número extraordinario, 213-234. Recuperado de http://www.revistaeducacion.educacion.es/re2008/re2008 09.pdf

- Montenegro, S. y Nodarse, F. (2017, julio-septiembre). La educación a distancia en entornos virtuales de enseñanza aprendizaje: Reflexiones didácticas, Atenas: Revista Científico Pedagógica, 3 (39), 31-47. Recuperado de http://atenas.umcc.cu/index.php/atenas/article/view/309/571

\section{CURRICULUM VITAE}

\section{Zoubair ACHARKI}

Profesor en el Departamento de Lengua y Literatura Hispánicas de la Facultad de Letras y Ciencias Humanas de la Universidad Abdelmalek Essaâdi de Tetuán. 